\title{
Fibro-Osseous Lesion Presenting with Refractory Microcytic
}

\section{Hypochromic Anemia}

\section{Iffat Jamal*}

Assistant Professor, Department of Hematology, Indira Gandhi Institute of Medical

Sciences, India

*Corresponding author: Iffat Jamal, Department of Hematology, Indira Gandhi

\section{Image Article}

Volume 3 Issue 2

Received Date: November 28, 2019

Published Date: December 04, 2019

Institute of Medical Sciences, Patna, India, Email: iffatjamal111@gmail.com

\section{Introduction}

Fibro-osseous lesions are benign tumors commonly presenting with multiple fractures, pain and limping [1]. Hematological manifestations are quite late and patients may present with microcytic hypochromic anemia, bicytopenia or pancytopenia [2]. A high index of clinical suspicion, radiological and hematological correlation is a must for early diagnosis and proper management [3-6].

A 21 year female patient presented with multiple fractures in both upper and lower limbs since birth. Her physical growth was retarted. Xray of pelvis and legs revealed multiple lytic lesions (Figure 1). Her vitamin D was $27.15 \mathrm{ng} / \mathrm{ml}$, Serum calcium was $9.9 \mathrm{mg} / \mathrm{dl}$ and serum Prolactin level was $4.83 \mathrm{ng} / \mathrm{ml}$ her hemogram revealed Hemoglobin of $8 \mathrm{gm} / \mathrm{dl}$, total leucocyte count of $9300 /$ cumm and platelet count of 2.3 lacs /cumm. On peripheral blood smear examination microcytic hypochromic anemia was found. Despite iron therapy patient was not responding to treatment. Hence bone marrow aspiration and biopsy was performed to find out the cause of resistant microcytic hypochromic anemia .Bone marrow was attempted thrice but didn't yield any diagnostic aspirate. Bone marrow biopsy revealed misshapen bony trabeculae focally rimmed by osteoblasts enclosing extensive fibro-collagenous stroma. Marked suppression of trilineage hematopoeisis was seen. A diagnosis of Fibro-osseous lesion was made favouring polyostotic fibrous dysplasia. Masson trichrome stain was done that showed extensive collagenisation in the intertrabecular spaces (Figures 2-4).

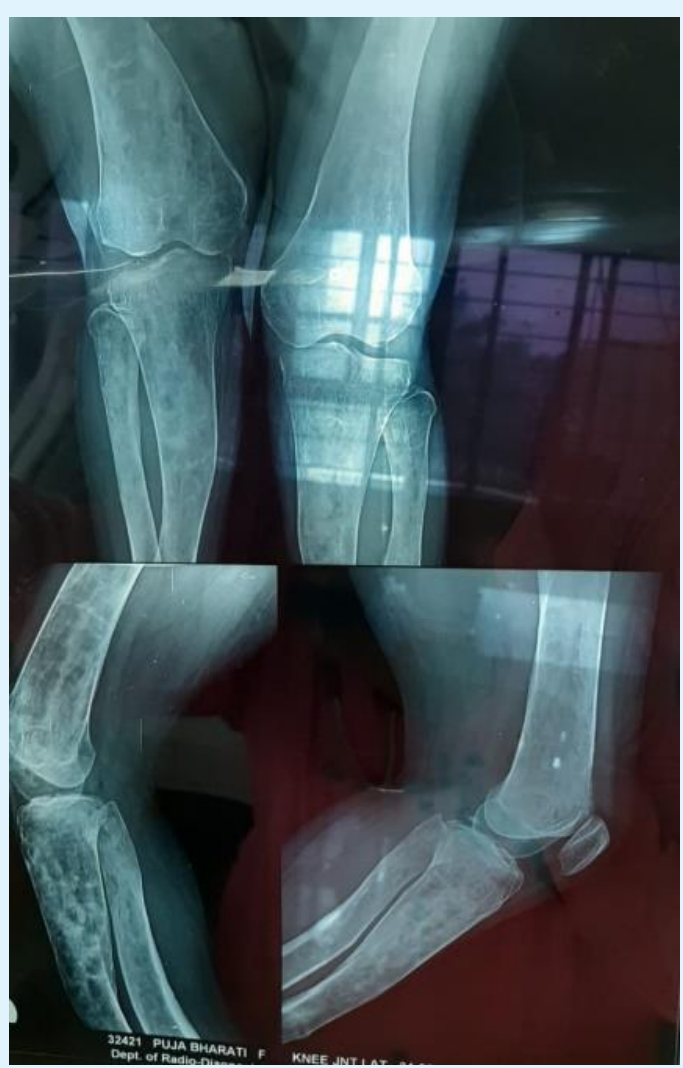

Figure 1: X-ray showing multiple lytic lesions in femur. 


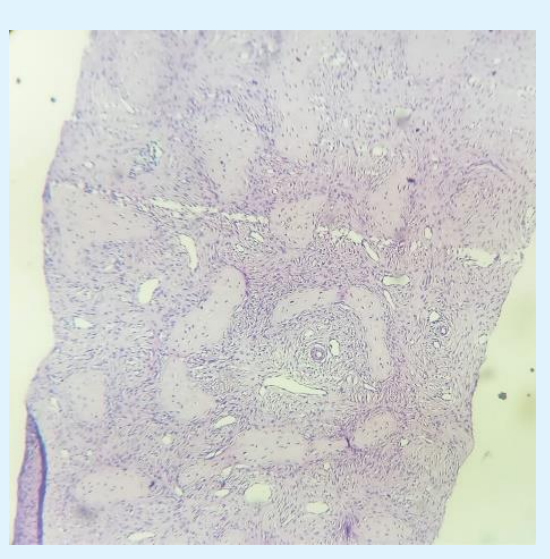

Figure 2: Microphotograph showing bony trabeculae enclosing fibrocollagenous stroma with absence of hematopoietic elements (H \& E; 100X).

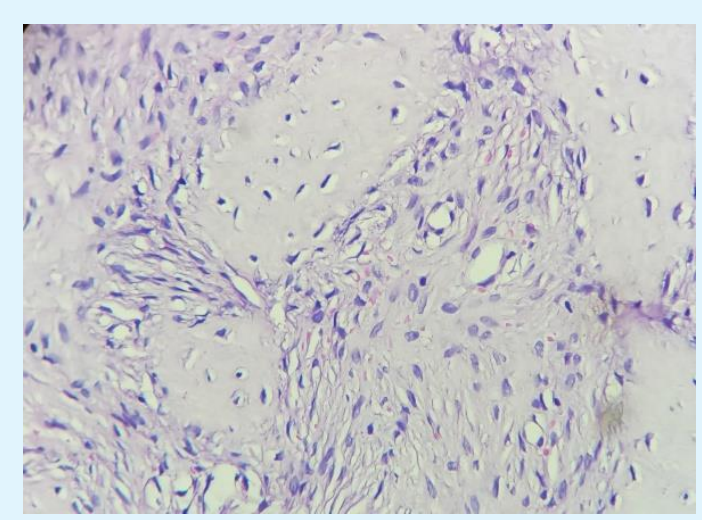

Figure 3: Microphotograph showing bony trabeculae focally rimmed by osteoblasts (H \& E; 400X).

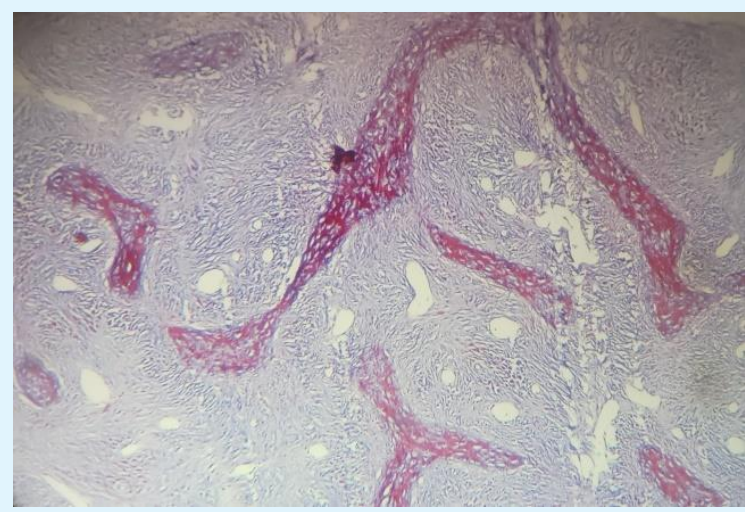

Figure 4: Microphotograph showing collagenisation in intertrabecular spaces (Masson's trichrome; 100X).

\section{References}

1. Farzaneh AH, Pardis PM (2005) Central giant cell granuloma and fibrous dysplasia occurring in the same jaw. Med Oral Patol Oral Cir Bucal 10(2): 130132.

2. Vegas Bustamante E, Gargallo Albiol J, Berini Ayt Ãs L, Gay Escoda C (2008) Benign fibro-osseous lesions of the maxillas: analysis of 11 cases. Med Oral Patol Oral Cir Bucal 13(10): 653-656.

3. Sankaranarayanan S, Srinivas $S$, Sivakumar $P$, Sudhakar R, Elangovan S (2011) "Hybrid" lesion of the maxilla. J Oral Maxillofac Pathol 15(3): 299-302.

4. Sontakke SA, Karjodkar FR, Umarji HR (2011) Computed tomographic features of fibrous dysplasia of maxillofacial region. Imaging Sci Dent 41(1): 23-28.

5. Chauhan I, Roy S, Garg V, Kavita M (2015) Fibroosseous lesions of the jaws: An insight. Int J Contemp Dent Med Rev pp: 1-5.

6. Srichinthu KK, Chitturi RT, Andamuthu Yamunadevi, Aparna Devi P, Deepak Ningombam S (2016) Fibro-osseous lesions-Classifications, pathophysiology and importance of radiology. a Short review Int Bio Biomed J Winter 2(1): 11-20. 